\title{
Light-curve and spectral properties of ultra-stripped core-collapse supernovae
}

\author{
Takashi J. Moriya
}

Division of Theoretical Astronomy, National Astronomical Observatory of Japan, National Institutes of Natural Sciences, 2-21-1 Osawa, Mitaka, Tokyo 181-8588, Japan email: takashi.moriya@nao.ac.jp

\begin{abstract}
We discuss light-curve and spectral properties of ultra-stripped core-collapse supernovae. Ultra-stripped supernovae are supernovae with ejecta masses of only $\sim 0.1 M_{\odot}$ whose progenitors lose their envelopes due to binary interactions with their compact companion stars. We follow the evolution of an ultra-stripped supernova progenitor until core collapse and perform explosive nucleosynthesis calculations. We then synthesize light curves and spectra of ultra-stripped supernovae based on the nucleosynthesis results. We show that ultra-stripped supernovae synthesize $\sim 0.01 M_{\odot}$ of the radioactive ${ }^{56} \mathrm{Ni}$, and their typical peak luminosity is around $10^{42} \mathrm{erg} \mathrm{s}^{-1}$ or $-16 \mathrm{mag}$. Their typical rise time is $5-10$ days. By comparing synthesized and observed spectra, we find that SN 2005ek and some of so-called calcium-rich gap transients like PTF10iuv may be related to ultra-stripped supernovae.
\end{abstract}

Keywords. supernovae: general, gravitational waves

Ultra-stripped supernovae (SNe) are SNe with ejecta masses of only $\sim 0.1 M_{\odot}$. When a SN progenitor has a compact companion, this kind of SNe with extreme stripping can occur (e.g., Tauris et al. 2013). We show light-curve and spectral properties of ultrastripped SNe in Fig. 1. See Moriya et al. (2016) for more details.
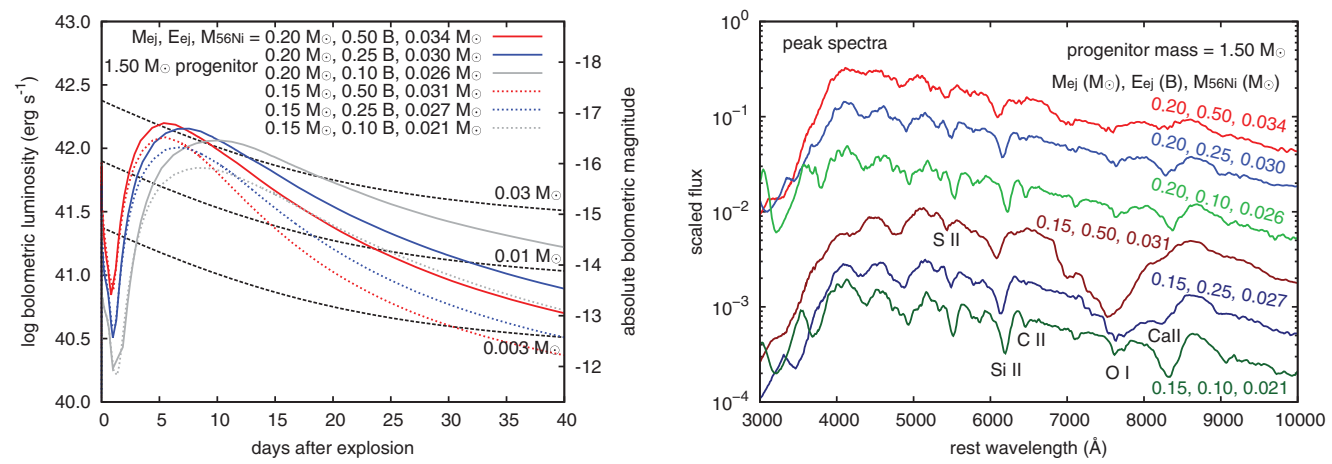

Figure 1. Light-curve (left) and spectral (right) properties of ultra-stripped SNe.

\section{References}

Moriya, T. J., Mazzali, P. A., Tominaga, N., Hachinger, S., Blinnikov, S. I., Tauris, T. M., Takahashi, K., Tanaka, M., Langer, N., \& Podsiadlowski, Ph. 2016, Mon. Not. R. Astron. Soc., accepted (arXiv:1612.02882)

Tauris, T. M., Langer, N., Moriya, T. J., Podsiadlowski, Ph., Yoon, S.-C., \& Blinnikov, S. I. 2013, Astrophys. J. Letters, 778, L23 